PREPARED FOR THE U.S. DEPARTMENT OF ENERGY, UNDER CONTRACT DE-AC02-76CH03073

PPPL-3772

PPPL-3772

UC-70

Pullback Transformations in Gyrokinetic Theory

by

H. Qin and W.M. Tang

January 2003

$\left.\stackrel{M}{M}\right|_{\substack{\text { PRInCETON PLASIMA } \\ \text { PHYSICS LABORATORY }}} ^{D}$

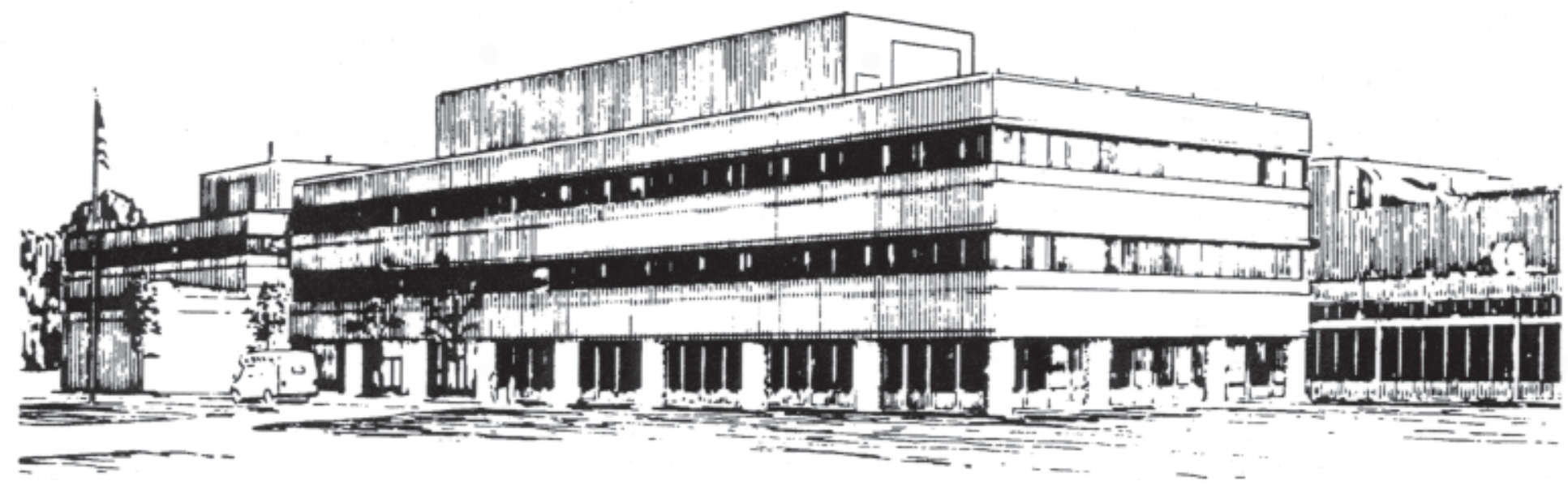

PRINCETON PLASMA PHYSICS LABORATORY PRINCETON UNIVERSITY, PRINCETON, NEW JERSEY 


\section{PPPL Reports Disclaimer}

This report was prepared as an account of work sponsored by an agency of the United States Government. Neither the United States Government nor any agency thereof, nor any of their employees, makes any warranty, express or implied, or assumes any legal liability or responsibility for the accuracy, completeness, or usefulness of any information, apparatus, product, or process disclosed, or represents that its use would not infringe privately owned rights. Reference herein to any specific commercial product, process, or service by trade name, trademark, manufacturer, or otherwise, does not necessarily constitute or imply its endorsement, recommendation, or favoring by the United States Government or any agency thereof. The views and opinions of authors expressed herein do not necessarily state or reflect those of the United States Government or any agency thereof.

\section{Availability}

This report is posted on the U.S. Department of Energy's Princeton Plasma Physics Laboratory Publications and Reports web site in Fiscal Year 2003. The home page for PPPL Reports and Publications is: http://www.pppl.gov/pub_report/

DOE and DOE Contractors can obtain copies of this report from:

U.S. Department of Energy

Office of Scientific and Technical Information

DOE Technical Information Services (DTIS)

P.O. Box 62

Oak Ridge, TN 37831

Telephone: (865) 576-8401

Fax: (865) 576-5728

Email: reports@adonis.osti.gov

This report is available to the general public from:

National Technical Information Service

U.S. Department of Commerce

5285 Port Royal Road

Springfield, VA 22161

Telephone: $1-800-553-6847$ or

(703) $605-6000$

Fax: (703) 321-8547

Internet: http://www.ntis.gov/ordering.htm 


\title{
Pullback Transformations in Gyrokinetic Theory
}

\author{
H. Qin and W. M. Tang \\ Plasma Physics Laboratory, Princeton University, Princeton, NJ 08543
}

\begin{abstract}
The Pullback transformation of the distribution function is a key component of the gyrokinetic theory. In this paper, a systematic treatment of this subject is presented, and results from applications of the uniform framework developed are reviewed. The focus is on providing a clear exposition of the basic formalism which arises from the existence of three distinct coordinate systems in gyrokinetic theory. The familiar gyrocenter coordinate system, where the gyromotion is decoupled from the rest of particle's dynamics, is non-canonical and non-fabric. On the other hand, Maxwell's equations, which are needed to complete a kinetic system, are initially only defined in the fabric laboratory phase space coordinate system. The pullback transformations provide a rigorous connection between the distribution functions in gyrocenter coordinates and Maxwell's equations in laboratory phase space coordinates. This involves the generalization of the usual moment integrals originally defined on the cotangent fiber of the phase space to the moment integrals on a general $6 \mathrm{D}$ symplectic manifold, is shown to be an important step in the proper formulation of gyrokinetic theory. The resultant systematic treatment of the moment integrals enabled by the pullback transformation. Without this vital element, a number of prominent physics features, such as the presence of the compressional Alfvén wave and a proper description of the gyrokinetic equilibrium, cannot be readily recovered.
\end{abstract}

PACS numbers: 52.25Fa, 52.25Dg, 52.35Py 


\section{INTRODUCTION}

Most of the interesting plasmas in laboratory and space are magnetized. The particle's motion in a magnetized equilibrium plasma consists of fast gyromotion and slow guiding center motion. It is the fast gyromotion which restricts the allowable time step in particle simulations of the associated dynamics in the laboratory phase space coordinate frame. In the past twenty years, gyrokinetic theory has been developed to remove the fast gyromotion from the kinetic system for low frequency and long parallel wavelength phenomena [1-13]. Gyrokinetic particle simulations, which use a much larger time step than the time scale of gyromotion [4, 14-20], have been successfully applied in studies of the transport problems of fusion plasmas. In particular, gyrokinetic theory offers a simplified version of the VlasovMaxwell system by utilizing the fact that in strongly magnetized plasmas the particle's gyroradius is much smaller than the scale length of the magnetic field: $\epsilon_{B} \equiv\left|\rho / L_{B}\right| \ll 1$, where $L_{B} \equiv|B / \nabla \boldsymbol{B}|$. More fundamentally, gyrokinetic theory requires the construction of a gyrocenter coordinate system in which the particle's gyromotion is decoupled from the rest of the particle dynamics. The Vlasov-Maxwell equation system can then be derived in this special coordinate system [21-26]. Guiding center coordinates are employed in the magnetostatic case, while gyrocenter coordinates are employed when there are electromagnetic perturbations in the system. Modern gyrokinetic theory [9-13, 21-26] utilizing non-canonical Hamiltonian and phase space Lie perturbation method [1-3] has been carefully established over a number of years. It not only sets up a rigorous and systematic foundation for the gyrokinetic framework, but also clarifies numerous confusing concepts and introduces much more physics content into the theory. For example, gyrokinetic theory has been extended to arbitrary frequency, arbitrary wavelength, electromagnetic perturbations in general geometries $[21-26]$.

One of the key components of modern gyrokinetic theory is the pullback transformation of the distribution function. This is responsible for many important physics properties in gyrokinetic theory. Famous examples include the polarization drift density in the gyrokinetic 
Poisson equation [4] and the polarization current in the gyrokinetic Ampere's law which accounts for the compressional Alfvén wave [23]. In this paper, a systematic treatment of this subject will be presented together with a brief summary of key results from applications of the uniform framework developed.

In Sec. II, the general theory of coordinate transformations and their pullback transformations in phase space are developed. In Sec. III, we investigate the physics of guiding center pullback transformation. The gyrocenter pullback transformation is studied in Sec. IV.

\section{COORDINATE TRANSFORMATIONS AND THEIR PULLBACK TRANS- FORMATIONS IN PHASE SPACE}

\section{A. Fabric Phase Space Coordinate System}

Physics is geometric and independent of coordinates, even though it can be more efficiently described with the help of coordinates. All possible choices of coordinate systems should be equivalent in terms of analyzing the physics of interest. If two coordinate systems are connected through a transformation, then the physics content is invariant with respect to the transformation. However, the mathematical involvement of different coordinate systems is indeed different when describing the same physics. For a given physics problem, the natural first step is to find the most efficient coordinate system. This can usually be constructed by imposing the desired mathematical structures. More often than not, it is constructed by perturbations around an obvious choice of coordinate system through a near identity coordinate transformation. In this sense, perturbation methods in physics are really about the quest of useful coordinates. In the present analysis, we will focus on the coordinate transformations in the $6 \mathrm{D}$ phase space (or the $8 \mathrm{D}$ extended phase space) for a single nonrelativistic classical particle. A coordinate transformation for the phase space $P$ of dimension 6 can be locally represented by a map between two subsets of the $R^{6}$ space, $T: \boldsymbol{x} \longmapsto \boldsymbol{X}=T(\boldsymbol{x})$. In the present study, we assume a coordinate transformation can be represented by a single 
map almost everywhere. The subset of coordinates which can not be covered by the single map has zero measure and does not contribute to the moment integrals.

Kinetic theory deals with particle distribution function $f$, which is a function defined on the phase space $P, f: P \rightarrow R$. In addition, kinetic theory in its common form implicitly makes use the fact that the phase space is the cotangent space (cotangent bundle) of a configuration space (manifold) $M, P=T^{*} M$. We call a coordinate system $(\boldsymbol{x}, \boldsymbol{v})$ a fiber coordinate system if $\boldsymbol{x}$ is the coordinate for $M$, and $\boldsymbol{v}$ is the coordinate for the cotangent fiber $T_{x}^{*} M$ at $\boldsymbol{x}$. A fiber coordinate system for the cotangent bundle of the laboratory configuration space will be referred to as the laboratory phase space coordinate system. The familiar moment integrals are actually fiber integrals of moment functions $q: P \rightarrow R$ performed on the cotangent fiber $T_{\boldsymbol{x}}^{*} M$ at each $\boldsymbol{x}$. In the laboratory phase space coordinate system $(\boldsymbol{x}, \boldsymbol{v})$, the moment integral of a moment function $q(\boldsymbol{x}, \boldsymbol{v})$ has the form

$$
q(\boldsymbol{x})=\int_{T_{\boldsymbol{x}}^{*} M} q(\boldsymbol{x}, \boldsymbol{v}) f(\boldsymbol{x}, \boldsymbol{v}) d^{3} \boldsymbol{v}
$$

The moment integrals yield results which are functions on the laboratory configuration space $M$. The moment integrals themselves are independent of the coordinate systems used for the cotangent fiber $T_{\boldsymbol{x}}^{*} M$ at each $\boldsymbol{x}$. In other words, $q(\boldsymbol{x})$ is invariant under a fiber coordinate transformation, i.e., a coordinate transformation that transforms only the fiber coordinate $\boldsymbol{v}$. Let $\varphi_{\boldsymbol{x}}: \boldsymbol{v} \rightarrow \boldsymbol{V}$ be a fiber coordinate transformation which in general depends on $\boldsymbol{x}$, the invariance of $q(\boldsymbol{x})$ can be expressed as

$$
q(\boldsymbol{x})=\int_{T_{\boldsymbol{x}}^{*} M} q(\boldsymbol{x}, \boldsymbol{v}) f(\boldsymbol{x}, \boldsymbol{v}) d^{3} \boldsymbol{v}=\int_{T_{\boldsymbol{x}}^{*} M} Q(\boldsymbol{x}, \boldsymbol{V}) F(\boldsymbol{x}, \boldsymbol{V}) d^{3} \boldsymbol{V}
$$

where $q(\boldsymbol{x}, \boldsymbol{v}) f(\boldsymbol{x}, \boldsymbol{v}) d^{3} \boldsymbol{v}$ and $Q(\boldsymbol{x}, \boldsymbol{V}) F(\boldsymbol{x}, \boldsymbol{V}) d^{3} \boldsymbol{V}$ are the representations of the same 3forms in $(\boldsymbol{x}, \boldsymbol{v})$ and $(\boldsymbol{x}, \boldsymbol{V})$ respectively. Here $d^{3} \boldsymbol{v}$ and $d^{3} \boldsymbol{V}$ can be viewed as the volume forms of $T_{\boldsymbol{x}}^{*} M$ at each $\boldsymbol{x}$. For example, in a magnetized plasma, the well-known $\left(v_{\|}, \mu, \theta\right)$ velocity coordinates, as well as the Cartesian velocity coordinates, can be used to calculate 
the same moment integrals,

$$
\begin{aligned}
q(\boldsymbol{x}) & =\int q\left(\boldsymbol{x}, v_{1}, v_{2}, v_{3}\right) f\left(\boldsymbol{x}, v_{1}, v_{2}, v_{3}\right) d v_{1} \wedge d v_{2} \wedge d v_{3} \\
& =\int \frac{B_{\|}}{m} Q\left(\boldsymbol{x}, v_{\|}, \mu, \phi\right) F\left(\boldsymbol{x}, v_{\|}, \mu, \phi\right) d v_{\|} \wedge d \mu \wedge d \theta
\end{aligned}
$$

In this example, the volume form $d v_{1} \wedge d v_{2} \wedge d v_{3}$ is canonical whereas the volume form $\left(B_{\|} / m\right) d v_{\|} \wedge d \mu \wedge d \theta$ is non-canonical. The most noticeable difference between them is that the former is a constant and the latter depends $\boldsymbol{x}$.

\section{B. Non-fabric Phase Space Coordinate System}

In gyrokinetic theory, however, useful coordinate systems are non-fabric. A non-fabric coordinate system $(\boldsymbol{X}, \boldsymbol{V})$ is a coordinate system where $\boldsymbol{X}$ is not necessarily the coordinates for the configuration space $M$, and $\boldsymbol{V}$ is not necessarily the coordinates for the cotangent fiber $T_{\boldsymbol{x}}^{*} M$ at each $\boldsymbol{x}$. A non-fabric coordinate transformation, by definition, transfers a fabric coordinate system into a non-fabric one. In the context of gyrokinetic theory, $(\boldsymbol{X}, \boldsymbol{V})$ can be either guiding center coordinates or the gyrocenter coordinates, both of which are non-fabric. The construction of the guiding center coordinates and the gyrocenter coordinates will be described in detail in Secs. III and IV. The discussion in this section applies to any general non-fabric coordinate systems and non-fabric transformations.

No matter which non-fabric coordinate system is used, the moment integrals are still defined on the cotangent fiber $T_{\boldsymbol{x}}^{*} M$ at each $\boldsymbol{x}$, and $q(\boldsymbol{x})$ should be invariant under such a general non-fabric coordinate transformation. For the new coordinate system $(\boldsymbol{X}, \boldsymbol{V})$ to be useful, it is necessary to know the construction of $q(\boldsymbol{x})$ in it. To be specific, the scenario studied in this paper is that the distribution function $f(\boldsymbol{x}, \boldsymbol{v})$ is known in the transformed non-fabric coordinate system $(\boldsymbol{X}, \boldsymbol{V})$ as $F(\boldsymbol{X}, \boldsymbol{V})$, whereas $q(\boldsymbol{x}, \boldsymbol{v})$ as a physical quantity, such as the position and the velocity, is only meaningfully defined in the laboratory phase space coordinate system $(\boldsymbol{x}, \boldsymbol{v})$. Given $q(\boldsymbol{x}, \boldsymbol{v})$ and $F(\boldsymbol{X}, \boldsymbol{V})$, there are two methods to cal- 
culate $q(\boldsymbol{x})$. The first method is to pull back the distribution function $F(\boldsymbol{X}, \boldsymbol{V})$ into $f(\boldsymbol{x}, \boldsymbol{v})$, and the second method depends on the generalization of the concept of moment integrals.

The first method, where the distribution function $F(\boldsymbol{X}, \boldsymbol{V})$ is pulled back into $f(\boldsymbol{x}, \boldsymbol{v})$, can be written as

$$
q(\boldsymbol{x})=\int_{T_{\boldsymbol{x}}^{*} M} q(\boldsymbol{x}, \boldsymbol{v}) \varphi^{*}[F(\boldsymbol{X}, \boldsymbol{V})] d^{3} \boldsymbol{v}
$$

where

$$
\varphi^{*}[F(\boldsymbol{X}, \boldsymbol{V})]=F(\boldsymbol{X}(\boldsymbol{x}, \boldsymbol{v}), \boldsymbol{V}(\boldsymbol{x}, \boldsymbol{v}))=f(\boldsymbol{x}, \boldsymbol{v})
$$

In the second method, we consider the generalization of the usual moment integrals originally defined on the cotangent fiber $T_{\boldsymbol{x}}^{*} M$ at each $\boldsymbol{x}$ to the moment integrals on a general 6 D symplectic manifold $P$. This can be accomplished by two different approaches. The first approach is to view $T_{\boldsymbol{x}}^{*} M$ as an orientable 3-subset of $P$ and $q(\boldsymbol{x})$ as an integral of a moment 3 -form $\lambda$ over such an 3-subset,

$$
q(\boldsymbol{x})=\int_{T_{\boldsymbol{x}}^{*} M} \lambda
$$

In the laboratory phase space coordinates $(\boldsymbol{x}, \boldsymbol{v})$, the moment 3 -form $\lambda$ is defined as

$$
\lambda=q(\boldsymbol{x}, \boldsymbol{v}) f(\boldsymbol{x}, \boldsymbol{v}) d^{3} \boldsymbol{v}
$$

where $d^{3} \boldsymbol{v}$ is the volume form for $T_{\boldsymbol{x}}^{*} M$ at a fixed $\boldsymbol{x}$. In a general non-fabric coordinate system $(\boldsymbol{X}, \boldsymbol{V})=\varphi(\boldsymbol{x}, \boldsymbol{v}), \lambda$ is pulled back from its form in $(\boldsymbol{x}, \boldsymbol{v})$,

$$
\Lambda=\varphi^{-1 *} \lambda=\varphi^{-1 *}\left[q(\boldsymbol{x}, \boldsymbol{v}) f(\boldsymbol{x}, \boldsymbol{v}) d^{3} \boldsymbol{v}\right]=\left[\varphi^{-1 *} q(\boldsymbol{x}, \boldsymbol{v})\right]\left[\varphi^{-1 *} f(\boldsymbol{x}, \boldsymbol{v})\right]\left[\varphi^{-1 *} d^{3} \boldsymbol{v}\right]
$$

where

$$
\begin{aligned}
& \varphi^{-1 *} q(\boldsymbol{x}, \boldsymbol{v})=Q(\boldsymbol{X}, \boldsymbol{V}) \equiv q(\boldsymbol{x}(\boldsymbol{X}, \boldsymbol{V}), \boldsymbol{v}(\boldsymbol{X}, \boldsymbol{V})) \\
& \varphi^{-1 *} f(\boldsymbol{x}, \boldsymbol{v})=F(\boldsymbol{X}, \boldsymbol{V}) \equiv f(\boldsymbol{x}(\boldsymbol{X}, \boldsymbol{V}), \boldsymbol{v}(\boldsymbol{X}, \boldsymbol{V}))
\end{aligned}
$$


Here we have assumed that the transformation $\varphi$ is a diffeomorphism (one-one onto and smooth), and $\varphi^{-1 *}$ is the pullback of $\varphi^{-1}$, which maps any function on $(\boldsymbol{x}, \boldsymbol{v})$ into a function on $(\boldsymbol{X}, \boldsymbol{V})$. Therefore, in a general non-fabric coordinate system $(\boldsymbol{X}, \boldsymbol{V}), q(\boldsymbol{x})$ can be expressed as

$$
\begin{aligned}
q(\boldsymbol{x}) & =\int_{T_{\boldsymbol{x}}^{*} M} \lambda=\int_{T_{\boldsymbol{x}}^{*} M} \varphi^{-1 *} \lambda=\int_{U=\{(\boldsymbol{X}, \boldsymbol{V}) \mid \boldsymbol{x}(\boldsymbol{X}, \boldsymbol{V})=\text { const. }\}} \Lambda \\
& =\int_{U=\{(\boldsymbol{X}, \boldsymbol{V}) \mid \boldsymbol{x}(\boldsymbol{X}, \boldsymbol{V})=\text { const. }\}}\left[\varphi^{-1 *} q(\boldsymbol{x}, \boldsymbol{v})\right] F(\boldsymbol{X}, \boldsymbol{V})\left[\varphi^{-1 *} d^{3} \boldsymbol{v}\right] .
\end{aligned}
$$

If the coordinates $\boldsymbol{v}=\left(v_{1}, v_{2}, v_{3}\right)$ for $T_{\boldsymbol{x}}^{*} M$ is canonical,

$$
d^{3} \boldsymbol{v}=d v_{1} \wedge d v_{2} \wedge d v_{3}
$$

then

$$
\varphi^{-1 *}\left[d^{3} \boldsymbol{v}\right]=d v_{1}(\boldsymbol{X}, \boldsymbol{V}) \wedge d v_{2}(\boldsymbol{X}, \boldsymbol{V}) \wedge d v_{3}(\boldsymbol{X}, \boldsymbol{V})
$$

There are practical difficulties associated with using Eq. (11) to calculate $q(\boldsymbol{x})$. First, the pullback of the volume form $\varphi^{-1 *} d^{3} \boldsymbol{v}$ has 15 terms in general, because the dimension of a general 3-form in the $6 \mathrm{D}$ phase space is 15 . Second, the integration domain expressed in the $(\boldsymbol{X}, \boldsymbol{V})$ coordinate system is complicated.

To get around these difficulties, the concept of a moment integral can be generalized by a different approach. Specifically, a moment integrals is generalized into a parameterized integral of a moment 6 -form $\lambda_{\boldsymbol{r}}$ in the $6 \mathrm{D}$ phase space

$$
\begin{aligned}
i(\boldsymbol{r}) & =\int \lambda_{\boldsymbol{r}}, \\
\lambda_{\boldsymbol{r}} & =i(\boldsymbol{r}, \boldsymbol{z}) f(\boldsymbol{z}) d^{6} \boldsymbol{z},
\end{aligned}
$$

where $\boldsymbol{z}=(\boldsymbol{x}, \boldsymbol{v})$ is phase space coordinate, $\boldsymbol{r}$ is a set of independent parameters, $i(\boldsymbol{r}, \boldsymbol{z})$ is a moment function of the phase space and the parameters $\boldsymbol{r}$, and $d^{6} \boldsymbol{z}$ is the Liouville volume 
from given by the symplectic structure $\omega$ in the phase space

$$
d^{6} \boldsymbol{z}=\frac{-1}{6 !} \omega \wedge \omega \wedge \omega \wedge \omega \wedge \omega \wedge \omega .
$$

In a canonical coordinate system

$$
\begin{aligned}
\omega & =\sum_{i=1}^{3} d x^{i} \wedge d v_{i} \\
d^{6} \boldsymbol{z} & =d x^{1} \wedge d x^{2} \wedge d x^{3} \wedge d v_{1} \wedge d v_{2} \wedge d v_{3} .
\end{aligned}
$$

Under a non-fabric coordinate transformation $\varphi, i(\boldsymbol{r})$ is obtained through the pullback of the $\boldsymbol{r}$-parameterized 6-form $\lambda_{\boldsymbol{r}}$,

$$
\begin{aligned}
i(\boldsymbol{r}) & =\int \varphi^{-1 *} \lambda_{\boldsymbol{r}}=\int \varphi^{-1 *}\left[i(\boldsymbol{r}, \boldsymbol{z}) f(\boldsymbol{z}) d^{6} \boldsymbol{z}\right] \\
& =\int \varphi^{-1 *}[i(\boldsymbol{r}, \boldsymbol{z}) f(\boldsymbol{z})] d^{6} \boldsymbol{Z}
\end{aligned}
$$

where $d^{6} \boldsymbol{Z}$ is the Liouville volume form of $(\boldsymbol{X}, \boldsymbol{V})$

$$
d^{6} \boldsymbol{Z}=\varphi^{-1 *} d^{6} \boldsymbol{z} .
$$

If $(\boldsymbol{x}, \boldsymbol{v})=\left(x_{1}, x_{2}, x_{3}, v_{1}, v_{2}, v_{3}\right)$ is a canonical coordinate system, $d^{6} \boldsymbol{Z}$ can be straightforwardly expressed as

$d^{6} \boldsymbol{Z}=\varphi^{-1 *} d^{6} \boldsymbol{z}=d x^{1}(\boldsymbol{X}, \boldsymbol{V}) \wedge d x^{2}(\boldsymbol{X}, \boldsymbol{V}) \wedge d x^{3}(\boldsymbol{X}, \boldsymbol{V}) \wedge d v_{1}(\boldsymbol{X}, \boldsymbol{V}) \wedge d v_{2}(\boldsymbol{X}, \boldsymbol{V}) \wedge d v_{3}(\boldsymbol{X}, \boldsymbol{V})$.

But in almost all the cases of practical interest, the Liouville volume form $d^{6} \boldsymbol{Z}=\varphi^{-1 *} d^{6} \boldsymbol{z}$ 
of $(\boldsymbol{X}, \boldsymbol{V})$ is more conveniently calculated through the pull-back of the symplectic structure

$$
\begin{aligned}
d^{6} \boldsymbol{Z} & =\frac{-1}{6 !} \Omega \wedge \Omega \wedge \Omega \wedge \Omega \wedge \Omega \wedge \Omega, \\
\Omega & =\varphi^{-1 *} \omega .
\end{aligned}
$$

One major difference between a canonical volume form and a non-canonical one is that the canonical volume form is a constant where the non-canonical volume form is generally a function of the phase space through its dependence on field variables. For the case of the gyrocenter coordinate system which will be discussed in Sec. IV, the volume form nontrivially depends on the perturbed electromagnetic fields. The usual moment integrals are special cases of the generalized moment integrals when

$$
i(\boldsymbol{r}, \boldsymbol{z})=\delta(\boldsymbol{x}-\boldsymbol{r}) q(\boldsymbol{z})
$$

That is

$$
q(\boldsymbol{r})=\int_{T_{r}^{*} M} q(\boldsymbol{r}, \boldsymbol{v}) f(\boldsymbol{r}, \boldsymbol{v}) d \boldsymbol{v}=\int \delta(\boldsymbol{x}-\boldsymbol{r}) q(\boldsymbol{x}, \boldsymbol{v}) f(\boldsymbol{x}, \boldsymbol{v}) d^{6} \boldsymbol{z} .
$$

From Eq. (18), the construction of $q(\boldsymbol{r})$ using the distribution function in the non-fabric coordinate system $F(\boldsymbol{X}, \boldsymbol{V})$ is

$$
\begin{aligned}
q(\boldsymbol{r}) & =\int \varphi^{-1 *}\left[\delta(\boldsymbol{x}-r) q(\boldsymbol{x}, \boldsymbol{v}) f(\boldsymbol{x}, \boldsymbol{v}) d^{6} \boldsymbol{z}\right] \\
& =\int \varphi^{-1 *}[\delta(\boldsymbol{x}-\boldsymbol{r})] \varphi^{-1 *}[q(\boldsymbol{x}, \boldsymbol{v})] \varphi^{-1 *}[f(\boldsymbol{x}, \boldsymbol{v})] d^{6} \boldsymbol{Z} \\
& =\int \delta(\boldsymbol{x}(\boldsymbol{X}, \boldsymbol{V})-\boldsymbol{r}) Q(\boldsymbol{X}, \boldsymbol{V}) F(\boldsymbol{X}, \boldsymbol{V}) d^{6} \boldsymbol{Z},
\end{aligned}
$$

Equation (4), (11), and (21) are equivalent and can be used interchangeably to simplify the calculation. In practice, the pull-backs involved are often associated with coordinate perturbation transformations, and thus can be further simplified by the perturbation techniques adopted. For example, in Eq. (21), the Liouville volume form $d^{6} \boldsymbol{Z}=\varphi^{-1 *} d^{6} \boldsymbol{z}$ of $(\boldsymbol{X}, \boldsymbol{V})$ will 
assume the same functional form as $d^{6} \boldsymbol{z}$ of $(\boldsymbol{x}, \boldsymbol{v})$ if $\varphi^{-1 *}$ preserves the symplectic structure of $(\boldsymbol{x}, \boldsymbol{v})$

$$
\Omega(\boldsymbol{Z})=\varphi^{-1 *} \omega(\boldsymbol{z})=[\omega(\boldsymbol{z})]_{\boldsymbol{z} \rightarrow \boldsymbol{Z}}
$$

Also, the term $\delta(\boldsymbol{x}(\boldsymbol{X}, \boldsymbol{V})-\boldsymbol{r})$ can be Taylor expanded in terms of the small perturbation parameter such that the integration in Eq. (21) can be carried out order by order. We will give such examples in Secs. III and IV in the context of the gyrokinetic theory.

\section{PULLBACK OF THE GUIDING CENTER COORDINATE TRANSFORMA- TION}

This section deals with the pull-back of the guiding center transformation, which is a necessary theoretical construction for analyzing the gyrokinetic equilibrium. The goal of the gyrokinetic equilibrium study is to understand magnetized plasmas in equilibrium using the guiding center coordinates. The gyrokinetic equilibrium is of fundamental importance for the widely adopted perturbative gyrokinetic particle simulation ( $\delta f$ method) [16-20], where the equilibrium distribution function and the electromagnetic field are assumed to be known. Gyrokinetic equilibria consistent with the well-studied fluid ones are obviously necessary for the perturbative gyrokinetic particle simulations to be reliable. In particular, recent numerical studies of equilibria with zonal flows [20] raise again the question of how to describe the equilibrium flow from the gyrokinetic point of view. The essence of the problem studied here is how to relate the measurable quantities in the laboratory frame to the information in the guiding center coordinates . Given a distribution function $F\left(\boldsymbol{X}, V_{\|}, \mu\right)$ in the guiding-center coordinates $\boldsymbol{Z}=\left(\boldsymbol{X}, V_{\|}, \mu, \xi\right)$, how are the fluid density, flow, and current calculated? Do the macroscopic field variables calculated from the gyrokinetic formalism satisfy the fluid equations obtained by taking the moments of the Vlasov equation in the laboratory phase space coordinates? The pullback formulas derived in Sec. II give answers to these questions, when applied to the guiding center transformation. 
For the current purpose, it is only necessary to know the leading order expression for the guiding center transformation $G: \boldsymbol{z} \mapsto \boldsymbol{Z}$, which transforms the laboratory phase space coordinates $\boldsymbol{z}=(\boldsymbol{x}, \boldsymbol{v})$ into the guiding center coordinates $\boldsymbol{Z}=\left(\boldsymbol{X}, V_{\|}, \mu, \xi\right)$,

$$
\begin{aligned}
\boldsymbol{X} & =\boldsymbol{x}-\boldsymbol{\rho}_{0}+O(\epsilon), \\
V_{\|} & =v_{\|}+O(\epsilon), \\
\mu & =\mu_{0}+O(\epsilon), \\
\xi & =\theta+O(\epsilon),
\end{aligned}
$$

where $\left(\boldsymbol{x}, v_{\|}, v_{\perp}, \theta\right)$ is the usual laboratory phase space coordinates. $\boldsymbol{\rho}_{0}$ and $\mu_{0}$, defined in particle coordinates, are the usual gyroradius and magnetic moment. $\theta$ is chosen such that $\hat{\boldsymbol{v}}_{\perp}=-e /|e|\left(\boldsymbol{e}_{x} \sin \theta+\boldsymbol{e}_{y} \cos \theta\right)$. $\boldsymbol{e}_{x}$ and $\boldsymbol{e}_{y}$ are two perpendicular directions in the configuration space, and $\left(\boldsymbol{e}_{x}, \boldsymbol{e}_{y}, \boldsymbol{b}\right)$ is a right-handed orthogonal frame. The guiding center coordinate system in a static magnetic field is illustrated in Figure 1. For the guiding center
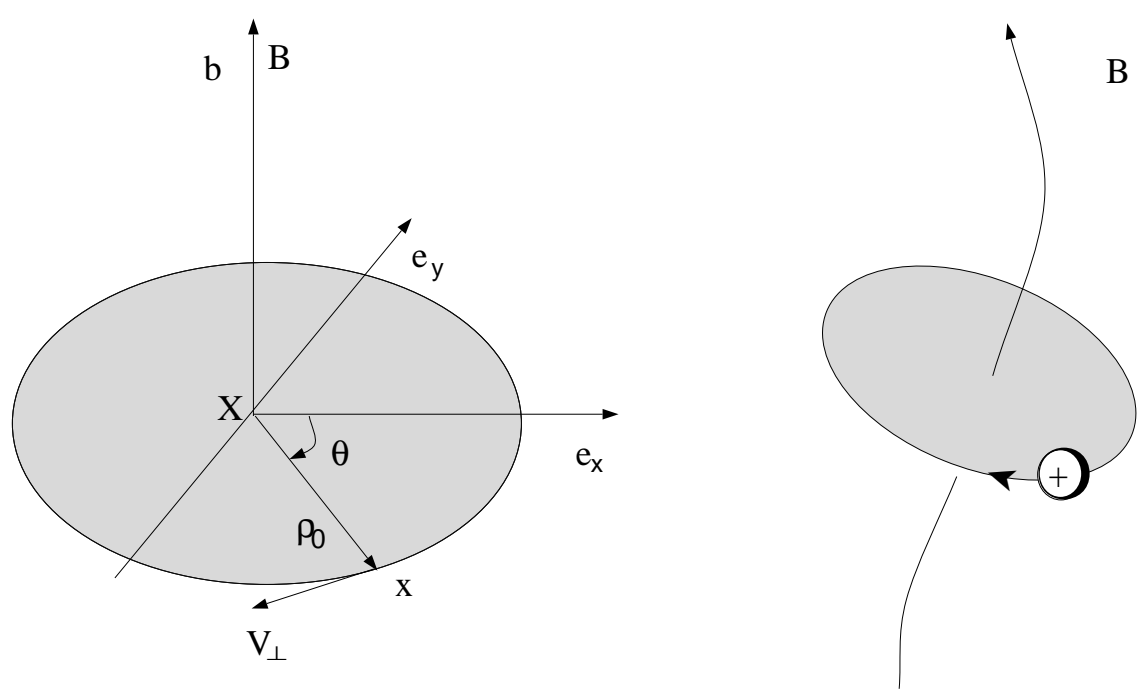

FIG. 1: Guiding Center Coordinate System 
transformation $G: \boldsymbol{z} \mapsto \boldsymbol{Z}$, Eq. (21) becomes

$$
\begin{aligned}
q(\boldsymbol{r}) & =\int q(\boldsymbol{r}, \boldsymbol{v}) f(\boldsymbol{r}, \boldsymbol{v}) d^{3} \boldsymbol{v}=\int q(\boldsymbol{z}) f(\boldsymbol{z}) \delta(\boldsymbol{x}-\boldsymbol{r}) d^{6} \boldsymbol{z} \\
& =\int G^{-1 *}[q(\boldsymbol{z}) \delta(\boldsymbol{x}-\boldsymbol{r})] F(\boldsymbol{Z}) d^{6} \boldsymbol{Z}=\int Q(\boldsymbol{Z}) \delta[\boldsymbol{X}+\boldsymbol{\rho}-\boldsymbol{r}] F(\boldsymbol{Z}) d^{6} \boldsymbol{Z},
\end{aligned}
$$

where it is assumed that the guiding center transformation $G$ is a diffeomorphism (one-one onto and smooth), and

$$
\begin{gathered}
d^{6} \boldsymbol{Z} \equiv B_{\|}^{*} / m d^{3} \boldsymbol{X} d V_{\|} d \mu d \xi \\
B_{\|}^{*}=\boldsymbol{b} \cdot \boldsymbol{B}^{*}, \quad \boldsymbol{B}^{*}=\boldsymbol{B}+\frac{c m V_{\|}}{e} \nabla \times \boldsymbol{b} . \\
Q(\boldsymbol{Z})=G^{-1 *} q(\boldsymbol{z}), \\
\boldsymbol{\rho}=G^{-1 *} \boldsymbol{\rho}_{0} .
\end{gathered}
$$

The physics encapsulated in the pull-back formula Eq.(26) is illustratively shown in Figure 2. An observable $q(\boldsymbol{r})$ at certain location $\boldsymbol{r}$ in the laboratory frame is the average of its microscopic counterpart expressed in the guiding center coordinates $Q(\boldsymbol{Z})$ over nearby guiding centers with $\boldsymbol{X}(\boldsymbol{Z})+\boldsymbol{\rho}(\boldsymbol{Z})=\boldsymbol{r}$. In Figure 2, three examples of such guiding centers are shown. For the number density in laboratory phase space coordinates, we use $q(\boldsymbol{z})=1$ and $G^{-1 *} 1=1$.

$$
\begin{aligned}
n(\boldsymbol{r}) & =\int \delta(\boldsymbol{X}+\boldsymbol{\rho}-\boldsymbol{r}) F(\boldsymbol{Z}) d^{6} \boldsymbol{Z}=\int \delta(\boldsymbol{X}-\boldsymbol{r}) F(\boldsymbol{Z}) d^{6} \boldsymbol{Z}+O\left(\epsilon^{2}\right) \\
& =2 \pi \int F(\boldsymbol{Z}) V_{\|} B_{\|}^{*} /\left.m d V_{\|} d \mu\right|_{\boldsymbol{X} \longmapsto \boldsymbol{r}}+O\left(\epsilon^{2}\right),
\end{aligned}
$$

where " $\left.\right|_{\boldsymbol{X} \longmapsto \boldsymbol{r}}$ " means replacing $\boldsymbol{X}$ by $\boldsymbol{r}$.

For the fluid velocity in laboratory phase space coordinates $\boldsymbol{u}(\boldsymbol{r})$, we have $q(\boldsymbol{z})=\boldsymbol{v}=\dot{\boldsymbol{x}}$, 


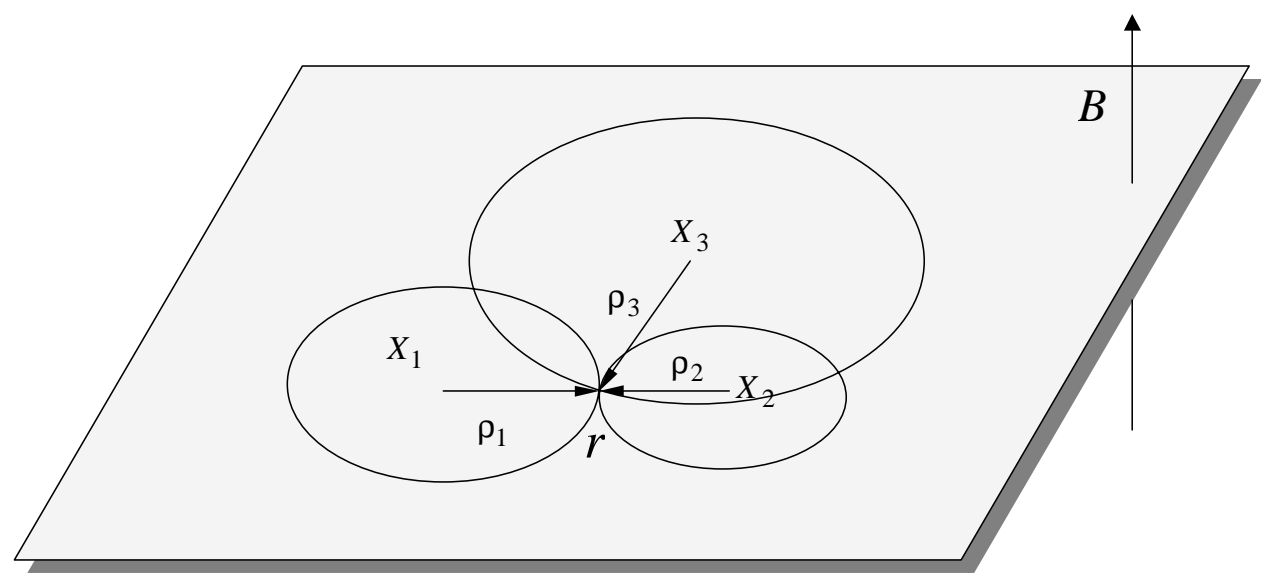

FIG. 2: The physics of pullback formula.

$G^{-1 *} \boldsymbol{v}=\dot{\boldsymbol{X}}+\dot{\boldsymbol{\rho}}(\boldsymbol{X})+O\left(\epsilon^{2}\right)$, and

$$
\begin{aligned}
\boldsymbol{u}(\boldsymbol{r}) & =\int(\dot{\boldsymbol{X}}+\dot{\boldsymbol{\rho}}) \delta(\boldsymbol{X}+\boldsymbol{\rho}-\boldsymbol{r}) F(\boldsymbol{Z}) d^{6} \boldsymbol{Z}+O\left(\epsilon^{2}\right) \\
& =\int\left[V_{\|} \boldsymbol{b}+\boldsymbol{V}_{\boldsymbol{E} \times \boldsymbol{B}}+\boldsymbol{V}_{d}\right] \delta(\boldsymbol{X}-\boldsymbol{r}) F(\boldsymbol{Z}) d^{6} \boldsymbol{Z} \\
& +\int \dot{\boldsymbol{\rho}} \delta(\boldsymbol{X}+\boldsymbol{\rho}-\boldsymbol{r}) F(\boldsymbol{Z}) d^{6} \boldsymbol{Z}+O\left(\epsilon^{2}\right) .
\end{aligned}
$$

The first term can be reduced to

$$
\begin{aligned}
\int\left[V_{\|} \boldsymbol{b}+\boldsymbol{V}_{\boldsymbol{E} \times \boldsymbol{B}}+\boldsymbol{V}_{d}\right] \delta(\boldsymbol{X}-\boldsymbol{r}) F(\boldsymbol{Z}) d^{6} \boldsymbol{Z}= \\
\left.\quad\left[n U_{\|} \boldsymbol{b}+\frac{c}{B} n \boldsymbol{E} \times \boldsymbol{b}+\frac{c}{e B} \boldsymbol{b} \times\left(W_{\perp} \frac{\nabla B}{B}+W_{\|} \boldsymbol{b} \cdot \nabla \boldsymbol{b}\right)\right]\right|_{\boldsymbol{X} \longmapsto \boldsymbol{r}}+O\left(\epsilon^{2}\right),
\end{aligned}
$$


where

$$
\begin{aligned}
U_{\|} & \equiv \frac{2 \pi}{n} \int V_{\|} B_{\|}^{*} / m F(\boldsymbol{Z}) d V_{\|} d \mu . \\
W_{\perp} & \equiv 2 \pi \int B \mu F(\boldsymbol{Z}) B_{\|}^{*} / m d V_{\|} d \mu, \\
W_{\|} & \equiv 2 \pi \int m V_{\|}^{2} F(\boldsymbol{Z}) B_{\|}^{*} / m d V_{\|} d \mu .
\end{aligned}
$$

The second term is the diamagnetic flow, which can be simplified in terms of $W_{\perp}$.

$$
\begin{aligned}
\int \dot{\boldsymbol{\rho}} \delta(\boldsymbol{X}+\boldsymbol{\rho}-\boldsymbol{r}) F(\boldsymbol{Z}) d^{6} \boldsymbol{Z} & =\int \dot{\boldsymbol{\rho} \rho} \cdot \nabla \delta(\boldsymbol{X}-\boldsymbol{r}) F(\boldsymbol{Z}) d^{6} \boldsymbol{Z}+O\left(\epsilon^{2}\right) \\
& =-\int \nabla \cdot\left[\boldsymbol{\rho} \dot{\boldsymbol{\rho}} B_{\|}^{*} / m F(\boldsymbol{Z})\right] \delta(\boldsymbol{X}-\boldsymbol{r}) d V_{\|} d \mu d \xi+O\left(\epsilon^{2}\right) \\
& =-\frac{c}{e} \nabla \times\left.\left(\boldsymbol{b} \frac{W_{\perp}}{B}\right)\right|_{\boldsymbol{X} \longmapsto \boldsymbol{r}}+O\left(\epsilon^{2}\right) .
\end{aligned}
$$

In Eq. (36), the following equations are used:

$$
\begin{aligned}
& \dot{\boldsymbol{\rho}}=\{\boldsymbol{\rho}, H\}=\sqrt{\frac{2 \mu B}{m}} \boldsymbol{e}_{\xi}+O(\epsilon), \\
& \left(\int \dot{\boldsymbol{\rho} \rho} d \xi\right)_{i j}=\frac{2 \pi \mu c}{e} \epsilon_{i j \boldsymbol{b}}+O(\epsilon) .
\end{aligned}
$$

Here, $\epsilon_{i j \boldsymbol{b}}$ is the Kronecker symbol, and the subscript $\boldsymbol{b}$ represents the dimension parallel to B.

Overall,

$$
\begin{aligned}
n(\boldsymbol{r}) \boldsymbol{u}(\boldsymbol{r}) & =\left[n U_{\|} \boldsymbol{b}+\frac{c \boldsymbol{b}}{e B} \times\left(W_{\perp} \nabla B+W_{\|} \boldsymbol{b} \cdot \nabla \boldsymbol{b}\right)\right. \\
& \left.+\frac{c n}{B} \boldsymbol{E} \times \boldsymbol{b}+\frac{c}{e} \nabla \times\left(\frac{W_{\perp}}{B} \boldsymbol{b}\right)\right]\left.\right|_{\boldsymbol{X} \longmapsto \boldsymbol{r}}+O\left(\epsilon^{2}\right) .
\end{aligned}
$$

It can be shown that the $n(\boldsymbol{r})$ and $n(\boldsymbol{r}) \boldsymbol{u}(\boldsymbol{r})$ derived above satisfy the usual fluid equations derived from the Vlasov equation in the laboratory phase space coordinate system by taking 
the velocity moments [25]. As an example, the equilibrium force balance equation can be recovered here. First, it is shown that

$$
\begin{gathered}
W_{\perp}(\boldsymbol{r})=2 \pi \int B \mu F(\boldsymbol{Z}) B_{\|}^{*} /\left.m d V_{\|} d \mu\right|_{\boldsymbol{X} \longmapsto \boldsymbol{r}} \\
=\int \frac{1}{2} m v_{\perp}^{2} f d^{3} \boldsymbol{v}+O(\epsilon)=p_{\perp}(\boldsymbol{r})+O(\epsilon), \\
W_{\|}\left((\boldsymbol{r})=2 \pi \int m V_{\|}^{2} F(\boldsymbol{Z}) B_{\|}^{*} /\left.m d V_{\|} d \mu\right|_{\boldsymbol{X} \longmapsto \boldsymbol{r}}\right. \\
=\left.\left[m n U_{\|}^{2}+2 \pi \int m\left(V_{\|}-U_{\|}\right)^{2} F(\boldsymbol{Z}) B_{\|}^{*} / m d V_{\|} d \mu\right]\right|_{\boldsymbol{X} \longmapsto \boldsymbol{r}} \\
=m n u_{\|}^{2}(\boldsymbol{r})+p_{\|}(\boldsymbol{r})+O(\epsilon) .
\end{gathered}
$$

From Equation (39),

$$
\begin{aligned}
n \boldsymbol{u}_{\perp} & =\left\{\frac{c \boldsymbol{b}}{e B} \times\left(W_{\perp} \nabla B+W_{\|} \boldsymbol{b} \cdot \nabla \boldsymbol{b}\right)\right. \\
& \left.+\frac{c n}{B} \boldsymbol{E} \times \boldsymbol{b}+\frac{c}{q}\left[\nabla \times\left(\frac{W_{\perp}}{B} \boldsymbol{b}\right)\right]_{\perp}\right\}\left.\right|_{\boldsymbol{X} \longmapsto \boldsymbol{r}}+O\left(\epsilon^{2}\right) \\
& =\frac{c}{e}\left\{-\frac{\nabla p_{\perp} \times \boldsymbol{b}}{B}+p_{\perp}\left[\frac{\boldsymbol{b} \times \nabla B}{B^{2}}-\left(\nabla \times \frac{\boldsymbol{b}}{B}\right)_{\perp}\right]+p_{\|} \frac{(\nabla \times \boldsymbol{b})_{\perp}}{B}\right. \\
& \left.+m n u_{\|}^{2} \frac{(\nabla \times \boldsymbol{b})_{\perp}}{B}\right\}+n \frac{\boldsymbol{E} \times \boldsymbol{b}}{B} c+O\left(\epsilon^{2}\right)
\end{aligned}
$$

Using

$$
\begin{aligned}
& (\boldsymbol{u} \cdot \nabla \boldsymbol{u}) \times \boldsymbol{b}=-u_{\|}^{2}(\nabla \times \boldsymbol{b})_{\perp}+O\left(\epsilon^{2}\right), \\
& \frac{\boldsymbol{b} \times \nabla B}{B^{2}}-\left(\nabla \times \frac{\boldsymbol{b}}{B}\right)_{\perp}=-\frac{(\nabla \times \boldsymbol{b})_{\perp}}{B},
\end{aligned}
$$


then leads to the result,

$$
n \boldsymbol{u}_{\perp}=-\frac{c}{e B}\left[m n \boldsymbol{u} \cdot \nabla \boldsymbol{u} \times \boldsymbol{b}+\nabla_{\perp} p_{\perp} \times \boldsymbol{b}-\left(p_{\|}-p_{\perp}\right)(\nabla \times \boldsymbol{b})_{\perp}-e n \boldsymbol{E} \times \boldsymbol{b}\right]+O\left(\epsilon^{2}\right),
$$

In a neutral plasma

$$
\boldsymbol{j}_{\perp}=\sum_{s}(e n \boldsymbol{u})_{s}=\frac{c}{B}\left[\boldsymbol{b} \times \nabla \sum_{s} p_{\perp}+\left(\sum_{s} p_{\|}-\sum_{s} p_{\perp}\right)(\nabla \times \boldsymbol{b})_{\perp}\right]
$$

This is the transverse equilibrium force balance equation. In particular, when the distribution function $F$ is isotropic, $\sum_{s} p_{\|}=\sum_{s} p_{\perp}=\sum_{s} p$, the familiar fluid result, $\boldsymbol{j}_{\perp}=c / B \boldsymbol{b} \times \nabla \sum_{s} p$, is recovered.

In the above derivation, the pullback formula has been used in the form of Eq. (21). The pullback formula in the form of Eq. (4) can be used to obtain the same results.

\section{PULLBACK OF THE GYROCENTER CENTER COORDINATE TRANS- FORMATION}

When time-dependent electromagnetic perturbations are introduced into a magnetized plasma, the guiding center coordinates used in Sec. III to study the gyrokinetic equilibrium will cease to be the "good" coordinate system where the gyromotion is decoupled from the rest of particle dynamics. To preserve the desirable decoupling of the gyromotion, a symplectic gyrocenter transformation can be constructed using a Lie perturbation method

$$
G y: \boldsymbol{Z}=(\boldsymbol{X}, U, \mu, \xi) \longmapsto \bar{Z}=(\overline{\boldsymbol{X}}, \bar{U}, \bar{\mu}, \bar{\xi})
$$

Due to the symplectic nature of the gyrocenter transformation, particle dynamics in the

gyrocenter coordinates are exactly the same as those in the guiding center coordinates except for a perturbation in the Hamiltonian. Since two consecutive coordinate transformations are 
involved, two pull-back transformations are needed to relate the distribution function in the gyrocenter coordinates $F_{G y}$ to the macroscopic physical quantities in the laboratory coordinates $q(\boldsymbol{r})$. In the guiding center coordinate $\boldsymbol{Z}=(\boldsymbol{X}, U, \mu, \xi)$,

$$
q(\boldsymbol{r})=\int\left[G^{-1 *} q\right](\boldsymbol{Z}) F(\boldsymbol{Z}) \delta\left(G^{-1} \boldsymbol{X}-\boldsymbol{r}\right) d^{6} \boldsymbol{Z}
$$

Replacing $F(\boldsymbol{Z})$ by its pull-back from the gyrocenter coordinate, we get,

$$
q(\boldsymbol{r})=\int\left[G^{-1 *} q\right](\boldsymbol{Z})\left[G y^{*} F_{G y}\right](\boldsymbol{Z}) \delta\left(G^{-1} \boldsymbol{X}-\boldsymbol{r}\right) d^{6} \boldsymbol{Z}
$$

In the above equations, $d^{6} \boldsymbol{Z}$ is understood to be $\left(B_{\|}^{*} / m\right) d^{3} \boldsymbol{X} d U d \mu d \xi$. Gy* is the pull-back transformation of the gyrocenter transformation, which transforms the distribution function in the gyrocenter coordinates into that in the guiding center coordinates. $G^{-1}$ is the inverse of $G$ that transforms the laboratory phase space coordinate system into the guiding center coordinates. It is assumed that the guiding center transformation $G$ and the gyrocenter transformation Gy are bijective and smooth. Note that in Eq. (49), Eq. (4) is used for the pull-back of the gyrocenter transformation Gy and Eq. (21) for the pullback of the guiding center transformation $G$.

The pull-back transformation from the gyrocenter coordinates to the guiding center coordinates is easily obtained from the expression for $G$ given by Ref. [11, 12, 21-23]. Since the focus of this paper is not the symplectic gyrocenter coordinate transformation, the expression for the pullback transformation is displayed in terms of the perturbed fields $\left(\boldsymbol{A}_{1}, \phi_{1}\right)$ without derivation,

$$
\begin{aligned}
& G y^{*} F=F+L_{\boldsymbol{G}} F=F-\frac{\boldsymbol{b}}{B} \times\left(\boldsymbol{A}_{1}+\frac{c}{e} \nabla S\right) \cdot \nabla F \\
& +\frac{e}{m c} \boldsymbol{b} \cdot\left(\boldsymbol{A}_{1}+\frac{c}{e} \nabla S\right) \frac{\partial F}{\partial U}+\frac{e}{m c}\left[\frac{e}{c} \boldsymbol{A}_{1} \cdot \frac{\partial \boldsymbol{\rho}}{\partial \xi}+\frac{\partial S}{\partial \xi}\right] \frac{\partial F}{\partial \mu}+O\left(\epsilon_{B}\right),
\end{aligned}
$$


where the gauge function $S$ satisfies

$$
\begin{aligned}
\left\{S, H_{0}\right\} & =\Omega \frac{\partial S}{\partial \bar{\xi}}+\frac{\partial S}{\partial t}+\frac{\partial S}{\partial \overline{\boldsymbol{X}}} \cdot\left\{\overline{\boldsymbol{X}}, H_{0}\right\}+\frac{\partial S}{\partial \bar{U}}\left\{\bar{U}, H_{0}\right\} \\
& =e \widetilde{\phi_{1}}(\overline{\boldsymbol{X}}+\boldsymbol{\rho}, t)-\frac{e}{c} \widetilde{\overline{\boldsymbol{V}} \cdot \boldsymbol{A}_{1}}(\overline{\boldsymbol{X}}+\boldsymbol{\rho}, t) .
\end{aligned}
$$

Here, $\widetilde{\phi}_{1}(\overline{\boldsymbol{X}}+\boldsymbol{\rho}, t)$ and $\widetilde{\overline{\boldsymbol{V}} \cdot \boldsymbol{A}_{1}}(\overline{\boldsymbol{X}}+\boldsymbol{\rho}, t)$ are the gyrophase dependent parts of $\phi_{1}(\overline{\boldsymbol{X}}+\boldsymbol{\rho}, t)$ and $\overline{\boldsymbol{V}} \cdot \boldsymbol{A}_{1}(\overline{\boldsymbol{X}}+\boldsymbol{\rho}, t)$ respectively,

$$
\begin{aligned}
\widetilde{\phi}_{1}(\overline{\boldsymbol{X}}+\boldsymbol{\rho}, t) & =\phi_{1}(\overline{\boldsymbol{X}}+\boldsymbol{\rho}, t)-\left\langle\phi_{1}(\overline{\boldsymbol{X}}+\boldsymbol{\rho}, t)\right\rangle \\
\widetilde{\overline{\boldsymbol{V}} \cdot \boldsymbol{A}_{1}}\left(\overline{\boldsymbol{X}}+\boldsymbol{\rho}_{0}, t\right) & =\overline{\boldsymbol{V}} \cdot \boldsymbol{A}_{1}(\overline{\boldsymbol{X}}+\boldsymbol{\rho}, t)-\left\langle\overline{\boldsymbol{V}} \cdot \boldsymbol{A}_{1}(\overline{\boldsymbol{X}}+\boldsymbol{\rho}, t)\right\rangle,
\end{aligned}
$$

and $H_{0}$ is the unperturbed Hamiltonian

$$
H_{0}=\frac{m \bar{U}^{2}}{2}+\bar{\mu} B
$$

In the coordinates $(\overline{\boldsymbol{X}}, \bar{U}, \bar{\mu}, \bar{\xi})$, the linear gyrokinetic equation is,

$$
\frac{\partial f}{\partial t}+\left(\bar{U} \boldsymbol{b}+\boldsymbol{v}_{d}\right) \cdot \nabla f-\frac{1}{m} \boldsymbol{b} \cdot \nabla H_{0} \frac{\partial f}{\partial \bar{U}}=\frac{c}{e B} \boldsymbol{b} \cdot\left(\nabla F_{0} \times \nabla H_{1}\right)-\frac{1}{m} \boldsymbol{b} \cdot\left(\nabla F_{0} \frac{\partial H_{1}}{\partial \bar{U}}-\nabla H_{1} \frac{\partial F_{0}}{\partial \bar{U}}\right),
$$

where

$$
\begin{aligned}
F & =F_{0}+f \\
H_{1} & =\left\langle e \phi_{1}(\overline{\boldsymbol{X}}+\boldsymbol{\rho}, t)-\frac{e}{c} \boldsymbol{V} \cdot \boldsymbol{A}_{1}(\overline{\boldsymbol{X}}+\boldsymbol{\rho}, t)\right\rangle .
\end{aligned}
$$

The importance of the pull-back formula in Eq. (49) is demonstrated in the following nontrivial examples.

In the following equations, $\boldsymbol{A}$ and $\phi$ will be used to represent the perturbed field. The subscript "1" will be dropped. Unless clarity requires the use of the barred notation, the 
bars for the gyrocenter coordinates will also be dropped.

\section{A. Gyrokinetic Shear Alfvén Wave}

For shear Alfvén physics,

$$
\boldsymbol{A}=A_{\|} \boldsymbol{b}
$$

The special form of Equation (51) for shear Alfvén modes is

$$
\Omega \frac{\partial S}{\partial \xi}=\frac{e}{\Omega}\left[\widetilde{\phi}(\boldsymbol{X}+\boldsymbol{\rho}, t)-\frac{1}{c} \widetilde{U A_{\|}}(\boldsymbol{X}+\boldsymbol{\rho}, t)\right] \approx \frac{e}{\Omega} \boldsymbol{\rho}_{0} \cdot\left[\nabla \phi(\boldsymbol{X}, t)-\frac{1}{c} U \nabla A_{\|}(\boldsymbol{X}, t)\right] .
$$

Using Eq. (50), we get the pull-back transformation for shear Alfvén modes,

$$
G y^{*} F=F+\frac{e}{m c} A_{\|}(\boldsymbol{X}+\boldsymbol{\rho}, t) \frac{\partial F}{\partial U}+\frac{e}{B}\left[\widetilde{\phi}(\boldsymbol{X}+\boldsymbol{\rho}, t)-\frac{1}{c} \widetilde{U A_{\|}}(\boldsymbol{X}+\boldsymbol{\rho}, t)\right] \frac{\partial F}{\partial \mu} .
$$

The perturbed density, perturbed flow, and perturbed current can be derived from the general form of Eq. (49).

$$
\begin{aligned}
n_{1}(\boldsymbol{r})= & \left\{\int\left[G y^{*}\left(F_{0}+f\right)\right](\boldsymbol{Z}) \delta(\boldsymbol{X}+\boldsymbol{\rho}-\boldsymbol{r}) d^{6} \boldsymbol{Z}\right\}_{1} \\
= & \int f(\boldsymbol{Z}) \delta(\boldsymbol{X}-\boldsymbol{r}) d^{6} \boldsymbol{Z}+\int[\delta(\boldsymbol{X}+\boldsymbol{\rho}-\boldsymbol{r})-\delta(\boldsymbol{X}-\boldsymbol{r})] f(\boldsymbol{Z}) d^{6} \boldsymbol{Z} \\
& +\int \delta(\boldsymbol{X}+\boldsymbol{\rho}-\boldsymbol{r})\left\{\frac{e}{m c} A_{\|}(\boldsymbol{X}+\boldsymbol{\rho}, t) \frac{\partial F_{0}}{\partial U}\right. \\
& \left.+\frac{e}{B}\left[\widetilde{\phi}(\boldsymbol{X}+\boldsymbol{\rho}, t)-\frac{1}{c} \widetilde{U A_{\|}}(\boldsymbol{X}+\boldsymbol{\rho}, t)\right] \frac{\partial F_{0}}{\partial \mu}\right\} d^{6} \boldsymbol{Z} .
\end{aligned}
$$

With regard to the physical meaning of this equation, the perturbed density in laboratory coordinates consists of three parts corresponding to the three integrals on the right hand side of the equation. The first integral is the (usual) perturbed density in gyrocenter coordinates, the second integral is the guiding center correction, and the third integral is the gyrocenter 
correction. After some lengthy algebra,

$$
n_{1}(\boldsymbol{r}, t)=\int J_{0} f(\boldsymbol{r}, U, \mu, t) d^{3} \boldsymbol{v}+\frac{e}{m} \nabla_{\perp} \frac{n_{0}}{\Omega^{2}} \nabla \phi(\boldsymbol{r}, t)+\frac{3}{4} \frac{e v_{t}^{2} n_{0}}{m \Omega^{4}} \nabla_{\perp}^{4} \phi(\boldsymbol{r}, t),
$$

where $d^{3} \boldsymbol{v}=2 \pi(B / m) d U d \mu, J_{0}=J_{0}\left(v_{\perp} \nabla_{\perp} / i \Omega\right)$ is the 0th order Bessel function of the first kind, and only terms up to $O\left(v_{\perp}^{4} \nabla_{\perp}^{4} / \Omega^{4}\right)$ for the $L_{G} F_{0}$ part of the pull-back transformation $G y^{*}$ have been retained. For the perturbed parallel flow,

$$
\begin{aligned}
n_{0} u_{\| 1}(\boldsymbol{r}) & =\left\{\int U\left[G y^{*}\left(F_{0}+f\right)\right](\boldsymbol{Z}) \delta(\boldsymbol{X}+\boldsymbol{\rho}-\boldsymbol{r}) d^{6} \boldsymbol{Z}\right\}_{1} \\
& =\int U f(\boldsymbol{Z}) \delta(\boldsymbol{X}-\boldsymbol{r}) d^{6} \boldsymbol{Z}+\int U[\delta(\boldsymbol{X}+\boldsymbol{\rho}-\boldsymbol{r})-\delta(\boldsymbol{X}-\boldsymbol{r})] f(\boldsymbol{Z}) d^{6} \boldsymbol{Z} \\
& +\int U \delta(\boldsymbol{X}+\boldsymbol{\rho}-\boldsymbol{r})\left\{\frac{e}{m c} A_{\|}(\boldsymbol{X}+\boldsymbol{\rho}, t) \frac{\partial F_{0}}{\partial U}\right. \\
& \left.+\frac{e}{B}\left[\widetilde{\phi}(\boldsymbol{X}+\boldsymbol{\rho}, t)-\frac{1}{c} \widetilde{U A_{\|}}(\boldsymbol{X}+\boldsymbol{\rho}, t)\right] \frac{\partial F_{0}}{\partial \mu}\right\} d^{6} \boldsymbol{Z} .
\end{aligned}
$$

Again, the algebra here is straightforward but involved. The final result is:

$n_{0} u_{\| 1}(\boldsymbol{r}, t)=\int J_{0} U f(\boldsymbol{r}, U, \mu, t) d^{3} \boldsymbol{v}+\int \frac{e}{m c}\left\langle U A_{\|}\left(\boldsymbol{r}+\boldsymbol{\rho}_{0}\right)\right\rangle \frac{\partial F_{0}}{\partial U} 2 \pi \frac{B}{m} d \mu d U+\frac{e n_{0} v_{t}^{2}}{2 m c \Omega^{2}} \nabla_{\perp}^{2} A_{\|}$,

where the first integral on the right hand side is the (usual) perturbed parallel flow of the gyrocenter, and the second integral and the third term are the gyrocenter correction generated by the pull-back transformation.

From Eq. (61), the quasi-neutrality condition is

$$
\sum_{j} e\left[\int J_{0} f d^{3} \boldsymbol{v}+\frac{e}{m} \nabla_{\perp} \frac{n_{0}}{\Omega^{2}} \nabla_{\perp} \phi+\frac{3 e}{4 m} \frac{v_{t}^{2}}{\Omega^{2}} \frac{n_{0}}{\Omega^{2}} \nabla_{\perp}^{4} \phi\right]=0 .
$$


From Eq. (63), the parallel Ampere's law is

$$
[\nabla \times \nabla \times \boldsymbol{A}]_{\|}=\frac{4 \pi}{c} \sum_{j} e \int\left(U J_{0} f+\frac{\partial F_{0}}{\partial U} \frac{e}{m c}\left\langle U A_{\|}\right\rangle\right) d^{3} \boldsymbol{v}+\frac{4 \pi}{c} \frac{e^{2} n_{0} v_{t}^{2}}{2 m c \Omega^{2}} \nabla_{\perp}^{2} A_{\|}
$$

In Eqs. (64) and (65), the spatial variable is the laboratory coordinate $\boldsymbol{r}$. However $\boldsymbol{r}$ is a dummy variable. What matters is the functional forms. We can replace $\boldsymbol{r}$ by the spatial coordinate of the gyrocenter coordinates $\boldsymbol{Z}$. Equations (64) and (65) will be referred to as the gyrokinetic quasineutrality condition and the gyrokinetic parallel Ampere's law, respectively.

As a simple application of these results, we derive the local dispersion relation in an unsheared slab geometry with $\boldsymbol{B}_{0}=B(x) \boldsymbol{e}_{z}$ and $n_{0}=n_{0}(x)$. For local perturbations

$$
\left(\phi, \psi_{\|}\right) \sim \mathrm{e}^{i\left(k_{y} y+k_{\|} z\right)}
$$

where $\psi_{\|}$is defined through

$$
A_{\|}=\frac{c}{i \omega}\left(\nabla \psi_{\|}\right)_{\|}
$$

The solution of the gyrokinetic equation, Eq. (54), for shear Alfvén waves in slab geometry is:

$$
f=-\frac{e}{T} F_{0}\left(\phi-\frac{k_{\|} U}{\omega} \psi_{\|}\right)+\frac{e}{T} \frac{\omega-\omega_{*}}{\omega-k_{\|} U} F_{0}\left(\phi-\frac{k_{\|} U}{\omega} \psi_{\|}\right),
$$

where $\omega_{*}$ is the diamagnetic drift frequency defined by

$$
\omega_{* j} \equiv\left(\frac{c T k_{y}}{L_{n} e B}\right)_{j}, \quad L_{n} \equiv\left(\frac{d \ln n}{d x}\right)^{-1},
$$

and the temperature gradient has been neglected. Substituting $f$ into the quasineutrality 
condition, we have,

$$
\begin{aligned}
& -\sum_{j} \frac{e^{2}}{m} \nabla_{\perp} \frac{n_{0}}{\Omega^{2}} \nabla_{\perp} \phi=-\sum_{j} \frac{e^{2} n_{0}}{T}[1+\zeta Z(\zeta)]\left(\phi-\psi_{\|}\right) \\
& +\sum_{j} \frac{e^{2} n_{0}}{T} \zeta Z(\zeta) \frac{\omega_{*}}{\omega}\left(\phi-\psi_{\|}\right)-\sum_{j} \frac{e^{2} n_{0}}{T} \frac{\omega_{*}}{\omega} \psi_{\|} .
\end{aligned}
$$

$Z(\zeta)$ is the plasma dispersion function and $\zeta \equiv \frac{\omega}{k_{\|} v_{t h}}$. Straightforward algebra shows that the parallel Ampere's law reduces to

$$
k_{\|}^{2} \psi_{\|}=\frac{\omega^{2}}{v_{A}^{2}} \phi,
$$

or in terms of $\omega_{A} \equiv k_{\|} v_{A}$,

$$
\psi_{\|}=\frac{\omega^{2}}{\omega_{A}^{2}} \phi .
$$

Inserting this polarization property into the quasineutrality condition, we obtain the desired dispersion relation,

$$
\begin{aligned}
& \sum_{j} \frac{e^{2} n_{0}}{m \Omega^{2}} k_{y}^{2}=-\sum_{j} \frac{e^{2} n_{0}}{T}[1+\zeta Z(\zeta)]\left(1-\frac{\omega^{2}}{\omega_{A}^{2}}\right) \\
& +\sum_{j} \frac{e^{2} n_{0}}{T} \zeta Z(\zeta) \frac{\omega_{*}}{\omega}\left(1-\frac{\omega^{2}}{\omega_{A}^{2}}\right)-\sum_{j} \frac{e^{2} n_{0}}{T} \frac{\omega_{*}}{\omega} \frac{\omega^{2}}{\omega_{A}^{2}} .
\end{aligned}
$$

It contains a large number of interesting physics effects for various parametric regimes. Some, which are relevant to tokamak plasmas, will be highlighted in the following discussion.

The fluid results are generally recovered from kinetic theory by ignoring the kinetic resonances and assuming the so-called "hot electron, cold ion expansion", that is,

$$
\begin{gathered}
\zeta_{e}=\frac{\omega}{v_{t h e} k_{\|}} \ll 1, \\
\zeta_{i}=\frac{\omega}{v_{t h i} k_{\|}} \gg 1 .
\end{gathered}
$$


Using the Taylor expansion and the asymptotic form for $Z(\zeta)$, gives:

$$
\begin{aligned}
& \frac{n_{0} m_{i} c^{2}}{B^{2}} k_{y}^{2}=\left[-\frac{e^{2} n_{e 0}}{T_{e}}+\frac{e_{i}^{2} n_{i 0}}{T_{i}}\left(\frac{v_{t h i} k_{\|}}{\omega}\right)^{2}\right]\left(1-\frac{\omega^{2}}{\omega_{A}^{2}}\right) \\
& -\frac{e_{i}^{2} n_{0 i}}{T_{i}} \frac{\omega_{* i}}{\omega}\left(1-\frac{\omega^{2}}{\omega_{A}^{2}}\right)-\left[\frac{e^{2} n_{e 0}^{2}}{T_{e}} \frac{\omega_{* e}}{\omega}+\frac{e_{i}^{2} n_{i 0}^{2}}{T_{i}} \frac{\omega_{* i}}{\omega}\right] \frac{\omega^{2}}{\omega_{A}^{2}} \\
& =\frac{e^{2} n_{e}}{T_{e}}\left[-1+\frac{e_{j} T_{e}}{|e| T_{i}}\left(\frac{v_{t h i} k_{\|}}{\omega}\right)^{2}+\frac{\omega_{* e}}{\omega}\right]\left[1-\frac{\omega^{2}}{\omega_{A}^{2}}\right] .
\end{aligned}
$$

Note the following relationship between $\omega_{* j}$ and $\omega_{* e}$ has been used,

$$
\omega_{* j}=-\frac{|e| T_{j}}{e_{j} T_{e}} \omega_{* e} .
$$

This then leads to the familiar fluid result [27]:

$$
\frac{e_{i} c_{s}^{2}}{|e| \Omega_{i}^{2}} k_{\perp}^{2}=\left[\frac{e_{i} c_{s}^{2}}{|e| \omega^{2}} k_{\|}^{2}-1+\frac{\omega_{* e}}{\omega}\right]\left[1-\frac{\omega^{2}}{\omega_{A}^{2}}\right],
$$

where

$$
c_{s}^{2} \equiv \frac{T_{e}}{m_{i}}
$$

It is commonly believed that when the plasma $\beta$ (ratio of plasma to magnetic pressure) approaches zero, the magnetic perturbations are not important. However, it is not a correct conclusion that, when $\beta$ goes to zero, there are no magnetic perturbations. Very obvious examples are the well-known shear Alfvén wave and the compressional Alfvén wave in a homogeneous magnetized plasma. Even in a zero $\beta$ magnetized plasma, the shear Alfvén wave and the compressional Alfvén wave are both mathematically and physically well-defined. The physical mechanism driving these two waves is the balance between plasma kinetic energy and the restoring force due to the bending or compression of the equilibrium magnetic field. Mathematically, they are characterized by the dispersion relations $\omega^{2}=k_{\|}^{2} v_{A}^{2}$ and $\omega^{2}=k^{2} v_{A}^{2}$ respectively. Their existence is independent of the plasma $\beta$.

This fact can also be verified from the dispersion relation, Eq. (70). When $\omega_{A} \gg \omega_{* e}$, 
there exists a solution in the range of $\omega_{A}$. For this range of $\omega$, the dispersion relation is reduced to

$$
\frac{n_{0} m_{i} c^{2}}{B^{2}} k_{y}^{2}=-\sum_{j} \frac{e^{2} n_{0}}{T}[1+\zeta Z(\zeta)]\left(1-\frac{\omega^{2}}{\omega_{A}^{2}}\right) .
$$

Under normal condition, term $\frac{n_{0} m_{i} c^{2}}{B^{2}} k_{y}^{2}$ is smaller by $O\left(\rho_{s}^{2} k_{\perp}^{2}\right)$, compared with the other terms. To the leading order,

$$
\omega^{2}=\omega_{A}^{2}
$$

The fact that there are no assumptions about $\zeta_{e}$ and $\zeta_{i}$ needed here to obtain this shear Alfvén wave is consistent with the basic physical picture of the shear Alfvén wave. The statement that when the plasma $\beta$ is small, magnetic perturbations are not important is always relevant only for a special class of electrostatic modes. In particular, for the electrostatic drift waves, magnetic perturbations are decoupled from these electrostatic perturbations when $\beta$ is small. This is evident from the fact that

$$
\frac{\omega_{* e}}{\omega_{A}} \rightarrow 0 \quad \text { as } \beta \rightarrow 0
$$

It is also evident from the polarization property $\psi_{\|}=\frac{\omega}{\omega_{A}} \phi$. For the electrostatic drift wave, $\omega \sim \omega_{* e}$,

$$
\psi_{\|}=\frac{\omega_{* e}}{\omega_{A}} \phi \rightarrow 0 \quad \text { as } \beta \rightarrow 0 .
$$

For the shear Alfvén branch, $\omega \sim \omega_{A}$,

$$
\psi_{\|} \sim \phi \quad \text { independent of } \beta
$$

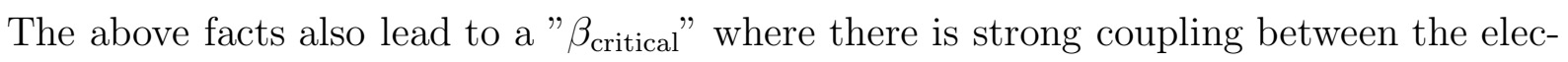
trostatic drift branch and the electromagnetic shear Alfvén branch. The criteria is $\omega_{* e} \sim \omega_{A}$. In tokamak geometry, it is [27],

$$
\sqrt{\beta_{\text {critical }}} \sim \frac{r}{R_{0}} \frac{r}{\rho_{s}}
$$


For standard tokamak parameters, $\beta_{\text {critical }}$ is not a very small number. However, $\omega_{A}$ is geometry dependent in complex geometries. It can be reduced to be as small as $\omega_{* e}$ even at low $\beta$. Another interesting limit is where $k_{\|}$approaches zero around mode rational surfaces, so that $\omega_{A}$ could be much smaller than its normal characteristic value. In both cases, there would be strong coupling between the shear Alfvén branch and the drift branch.

\section{B. Compressional Alfvén Wave}

In this subsection, the simplest example of the compressional Alfvén wave in a homogeneous magnetized plasma is used to demonstrate the essence of the pull-back formula in the perpendicular direction. $\boldsymbol{B}_{0}$ is assumed to be in the $\boldsymbol{e}_{z}$ direction, and for simplicity, we let

$\boldsymbol{k}=k_{y} \boldsymbol{e}_{y}$. The MHD results for the compressional Alfvén wave indicate that the magnetic perturbation is in the parallel direction, the electrical perturbation and current perturbation are in the $\boldsymbol{e}_{x}$ direction, and the plasma displacement is in the $\boldsymbol{e}_{y}$ direction. From the kinetic point of view, we can choose

$$
\phi=0 \quad \text { and } \quad \boldsymbol{A}=A_{x} \boldsymbol{e}_{x} .
$$

The gyrokinetic equation is:

$$
\frac{\partial f}{\partial t}+U \boldsymbol{b} \cdot \nabla f=0
$$

Assuming $A_{x}, f \propto \mathrm{e}^{i k_{y} y-i \omega t}$, then leads to $f=0$.

Interesting physics is found in the gyrocenter pull-back transformation. In order to obtain the necessary pull-back transformation to $O\left(\epsilon_{\omega}^{2}\right)$, where $\epsilon_{\omega} \equiv \omega / \Omega \ll 1$, we need to solve for $\partial S / \partial \xi$ from Eq. (51). Let

$$
S=S^{(0)}+\epsilon_{\omega} S^{(1)}+\epsilon_{\omega}^{2} S^{(2)}+O\left(\epsilon_{\omega}^{3}\right)
$$


To the 3rd order, the solution for $S$ is

$$
\begin{aligned}
\Omega \frac{\partial S}{\partial \xi} \approx \Omega & \frac{\partial S^{(0)}+S^{(1)}+S^{(2)}}{\partial \xi}+O\left(\epsilon_{\omega}^{3}\right) \\
= & e\left(\widetilde{\phi}-\frac{1}{c} \widetilde{\boldsymbol{V} \cdot \boldsymbol{A}}\right)-\frac{e}{\Omega} \frac{\partial}{\partial t} \int\left(\widetilde{\phi}-\frac{1}{c} \widetilde{\boldsymbol{V} \cdot \boldsymbol{A}}\right) d \xi \\
& +\frac{e}{\Omega^{2}} \frac{\partial^{2}}{\partial t^{2}} \iint\left(\widetilde{\phi}-\frac{1}{c} \widetilde{\boldsymbol{V} \cdot \boldsymbol{A}}\right) d \xi d \xi+O\left(\epsilon_{\omega}^{3}\right) .
\end{aligned}
$$

From the general form of the gyrocenter pull-back transformation we have:

$$
\begin{aligned}
{\left[G y^{*}\left(F_{0}+f\right)\right]_{1} } & =f+\frac{e}{m c} \frac{\partial F_{0}}{\partial \mu}\left\{-\frac{e v_{\perp}^{2}}{\Omega^{2} c} B_{\|}\right. \\
& \left.-\frac{e}{\Omega^{2}} \frac{\partial}{\partial t} \int\left(\widetilde{\phi}-\frac{1}{c} \widetilde{\boldsymbol{V} \cdot \boldsymbol{A}}\right) d \xi+\frac{e}{\Omega^{3}} \frac{\partial}{\partial t^{2}} \iint\left(\widetilde{\phi}-\frac{1}{c} \widetilde{\boldsymbol{V} \cdot \boldsymbol{A}}\right) d \xi d \xi\right\} .
\end{aligned}
$$

In above derivation, we have used the following expressions for the gyro-average:

$$
\begin{aligned}
H_{1} & =\left\langle e \phi(\boldsymbol{X}+\boldsymbol{\rho}, t)-\frac{e}{c} \boldsymbol{V} \cdot \boldsymbol{A}(\boldsymbol{X}+\boldsymbol{\rho}, t)\right\rangle \\
& \approx e\left[\phi(\boldsymbol{X}, t)-\frac{1}{c} U A_{\|}(\boldsymbol{X}, t)+\frac{v_{\perp}^{2}}{c \Omega} B_{\|}(\boldsymbol{X}, t)\right],
\end{aligned}
$$

The perpendicular Ampere's law is needed to complete this system of equations. For this purpose, it is necessary to obtain the perturbed perpendicular current.

$$
\begin{aligned}
n_{0} \boldsymbol{u}_{\perp}= & \left\{\int \boldsymbol{V}_{\perp}\left[G y^{*}\left(F_{0}+f\right)\right](\boldsymbol{Z}) \delta(\boldsymbol{X}+\boldsymbol{\rho}-\boldsymbol{r}) d^{6} \boldsymbol{Z}\right\}_{1} \\
= & \int \boldsymbol{V}_{\perp} \delta(\boldsymbol{X}+\boldsymbol{\rho}-\boldsymbol{r}) f(\boldsymbol{Z}) d^{6} \boldsymbol{Z}+\int \boldsymbol{V}_{\perp} \delta(\boldsymbol{X}+\boldsymbol{\rho}-\boldsymbol{r}) \frac{e}{m c} \frac{\partial F_{0}}{\partial \mu}\left\{\frac{-e v_{\perp}^{2}}{\Omega^{2} c} B_{\|}\right. \\
& \left.-\frac{e}{\Omega^{2}} \frac{\partial}{\partial t} \int\left(\widetilde{\phi}-\frac{1}{c} \widetilde{\boldsymbol{V} \cdot \boldsymbol{A}}\right) d \xi+\frac{e}{\Omega^{3}} \frac{\partial}{\partial t^{2}} \iint\left(\widetilde{\phi}-\frac{1}{c} \widetilde{\boldsymbol{V} \cdot \boldsymbol{A}}\right) d \xi d \xi\right\} d^{6} \boldsymbol{Z} .
\end{aligned}
$$

FLR effects are ignored here, and use the following expression for the particle perpendicular velocity

$$
\boldsymbol{V}_{\perp}=-V_{\perp}\left[\sin (\xi) \boldsymbol{e}_{x}+\cos (\xi) \boldsymbol{e}_{y}\right],
$$


is utilized. Finally, the perturbed perpendicular flow is

$$
\begin{aligned}
n_{0} \boldsymbol{u}_{\perp} & =\int \boldsymbol{V}_{\perp} \delta(\boldsymbol{X}-\boldsymbol{r}) \frac{e}{m c} \frac{\partial F_{0}}{\partial \mu}\left\{\frac{e}{\Omega^{2}} \frac{\partial}{\partial t} \int \frac{1}{c} \boldsymbol{V}_{\perp} \cdot \boldsymbol{A}_{\perp} d \xi\right. \\
& \left.-\frac{e}{\Omega^{3}} \frac{\partial^{2}}{\partial t^{2}} \iint \frac{1}{c} \boldsymbol{V}_{\perp} \cdot \boldsymbol{A}_{\perp} d \xi d \xi\right\} d^{6} \boldsymbol{Z} \\
& =\frac{-i \omega n_{0} e^{2} B}{m^{2} c^{2} \Omega^{2}} A_{x} \boldsymbol{e}_{y}+\frac{\omega^{2} n_{0} e^{2} B}{m^{2} c^{2} \Omega^{3}} A_{x} \boldsymbol{e}_{x}=\frac{n_{0} c}{B^{2}} \boldsymbol{E} \times \boldsymbol{B}+\frac{n_{0} m c^{2}}{e B^{2}} \frac{\partial \boldsymbol{E}_{\perp}}{\partial t} .
\end{aligned}
$$

It is obvious that the $\boldsymbol{E} \times \boldsymbol{B}$ flow does not contribute to the perpendicular current, which is generated by the ion polarization drift,

$$
\boldsymbol{j}=\sum_{j}\left(e n_{0} \boldsymbol{u}_{\perp}\right)_{j} \approx \frac{n_{0} m_{i} c}{B^{2}} \omega^{2} A_{x} \boldsymbol{e}_{x} .
$$

The perpendicular Ampere's law $(\nabla \times \nabla \times \boldsymbol{A})_{\perp}=4 \pi / c \boldsymbol{j}_{\perp}$ gives:

$$
k_{y}^{2} A_{x}=\frac{4 \pi n_{0} m_{i}}{B^{2}} \omega^{2} A_{x}, \quad \text { or } \quad \omega^{2}=k_{y}^{2} v_{A}^{2} .
$$

This is the compressional Alfvén wave. The key element in this gyrokinetic description of the compressional Alfvén wave is the perturbed perpendicular current. To $O\left(\epsilon_{\omega}\right)$, the perpendicular flow is the $\boldsymbol{E} \times \boldsymbol{B}$ flow, which gives no current. Therefore, it is necessary to go to $O\left(\epsilon_{\omega}^{2}\right)$. The current to this order is the current generated by the polarization drift in the perturbed electromagnetic field.

\section{Bernstein Wave}

In this subsection, the Bernstein wave and is recovered, and the application of the pullback transformations to high frequency modes is demonstrated. We consider an electrostatic wave propagating in a homogeneous magnetized plasma with $\omega \sim \Omega$. Let $\boldsymbol{B}_{0}=B \boldsymbol{e}_{z}$ and 
$\boldsymbol{k}=k \boldsymbol{e}_{x}$. The solution for the linear gyrokinetic equation is degenerate because $k_{\|}=0$,

$$
f=-\frac{e}{T} F_{0} \frac{-k_{\|} U}{\omega-k_{\|} U} \phi=0
$$

As in the case of compressional Alfvén wave, $f$, the gyrophase independent part of the distribution function, does not play any role, and the only physics content is found in the pull-back of the perturbed density, which requires expressing the gauge function $S$ in terms of the perturbed fields. The equation for $S$ is

$$
\left\{S, H_{0}\right\}=\Omega \frac{\partial S}{\partial \xi}+\frac{\partial S}{\partial t}=e \widetilde{\phi}(\boldsymbol{X}+\boldsymbol{\rho})=e\left[\mathrm{e}^{\boldsymbol{\rho} \cdot \nabla}-J_{0}\left(\frac{\boldsymbol{\rho} \cdot \nabla}{i}\right)\right] \phi
$$

That is,

$$
\frac{\partial S}{\partial \xi}-i \bar{\omega} S=\frac{e}{\Omega}\left[\mathrm{e}^{i \rho k \cos \xi}-J_{0}(\rho k)\right] \phi,
$$

where $\bar{\omega}=\frac{\omega}{\Omega}$. Using the identity

$$
\mathrm{e}^{\lambda \cos \xi}=\sum_{n=-\infty}^{\infty} I_{n}(\lambda) \mathrm{e}^{i n \xi}
$$

we solve for $S$,

$$
S=\frac{e}{\Omega i \bar{\omega}} J_{0} \phi+\frac{e}{\Omega} \sum_{n=-\infty}^{\infty} \frac{I_{n}(i \rho k)}{i(n-\bar{\omega})} \mathrm{e}^{i n \xi} \phi .
$$

Only $\partial S / \partial \xi$ is needed in the pull-back transformation,

$$
\frac{\partial S}{\partial \xi}=\frac{e}{\Omega} \sum_{n=-\infty}^{\infty} \frac{n I_{n}(i \rho k)}{(n-\bar{\omega})} \mathrm{e}^{i n \xi} \phi
$$


The density response comes only from the pull-back transformation since $f=0$.

$$
\begin{aligned}
n_{1} & =\int J_{0} f d^{3} \boldsymbol{v}+\int \delta(\boldsymbol{X}+\boldsymbol{\rho}-\boldsymbol{r}) \frac{e}{m c} \frac{\partial S}{\partial \xi} \frac{\partial F_{0}}{\partial \mu} d^{6} \boldsymbol{Z} \\
& =\int\left[\mathrm{e}^{\boldsymbol{\rho} \cdot \nabla} \delta(\boldsymbol{X}-\boldsymbol{r})\right] \frac{-e}{T} F_{0} \sum_{n=-\infty}^{\infty} \frac{n I_{n}(i \rho k)}{(n-\bar{\omega})} \mathrm{e}^{i n \xi} \phi d^{6} \boldsymbol{Z} .
\end{aligned}
$$

Using the facts that

$$
\int\left[\mathrm{e}^{\rho \cdot \nabla} \delta(\boldsymbol{X}-\boldsymbol{r})\right] Q d^{6} \boldsymbol{Z}=\int \delta(\boldsymbol{X}-\boldsymbol{r}) \mathrm{e}^{-\boldsymbol{\rho} \cdot \nabla} Q d^{6} \boldsymbol{Z}
$$

and

$$
\int_{0}^{2 \pi} \mathrm{e}^{i(m+n) \xi} d \xi=\delta_{m,-n} 2 \pi
$$

we have

$$
n_{1}=\frac{2 \pi}{(2 \pi T / m)^{3 / 2}} \int \frac{-n_{0} e \phi}{T} \exp \left(-\frac{v_{\|}^{2}+v_{\perp}^{2}}{2 T / m}\right) \sum_{n=-\infty}^{\infty} \frac{n I_{-n}(-i \rho k) I_{n}(i \rho k)}{(n-\bar{\omega})} v_{\perp} d v_{\|} d v_{\perp} .
$$

The following properties of the Bessel functions are needed to finish the integral:

$$
\begin{aligned}
I_{n}(x) & =i^{-n} J_{n}(i x), \\
J_{-n}(x) & =(-1)^{n} J_{n}(x)=J_{n}(-x), \\
\int_{0}^{\infty} t \mathrm{e}^{-p t^{2}} J_{n}^{2}(a t) d t & =\frac{1}{2 p} \mathrm{e}^{-a^{2} / 2 p} I_{n}\left(\frac{a^{2}}{2 p}\right) .
\end{aligned}
$$

Carrying out the algebra, we obtain

$$
n_{1}=n_{0} \frac{e \phi}{T} \sum_{n=1}^{\infty} \frac{2 n^{2}}{\left(\frac{\omega}{\Omega}\right)^{2}-n^{2}} \exp \left(-\frac{k^{2} T}{\Omega^{2} m}\right) I_{n}\left(\frac{k^{2} T}{\Omega^{2} m}\right)
$$


Finally, the Poisson equation $-\nabla^{2} \phi=\sum_{j} 4 \pi\left(e n_{1}\right)_{j}$ gives the dispersion relation,

$$
1=\sum_{j} \frac{4 \pi n_{0} e^{2}}{T k^{2}} \sum_{n=1}^{\infty} \frac{2 n^{2}}{\left(\frac{\omega}{\Omega}\right)^{2}-n^{2}} \exp \left(-\frac{k^{2} T}{\Omega^{2} m}\right) I_{n}\left(\frac{k^{2} T}{\Omega^{2} m}\right)
$$

This is the Bernstein wave.

\section{CONCLUSIONS}

The pullback transformations of the phase space coordinate system transformations have been studied here in the context of gyrokinetic theory. The necessity of such a study arises from the existence of three different coordinate systems in the gyrokinetic theory. The familiar gyrocenter coordinate system, where the gyromotion is decoupled from the rest of particle's dynamics, is non-canonical and non-fabric. On the other hand, Maxwell's equations, which are needed to complete a kinetic system, are first only defined in the fabric laboratory phase space coordinate system. The pullback transformations are needed to connect the distribution functions in the gyrocenter coordinates and Maxwell's equations in the laboratory phase space coordinates. In order to gain a systematic understanding of the mathematical construction and physical implications of the pullback transformations, we have adopted a geometric (coordinate independent) viewpoint for the moment integrals originally defined in the laboratory phase space coordinate system. The moment integrals in kinetic theories are geometrically interpreted as integrals of 3-forms over a 3-subset of the phase-space. Therefore, they are independent of the coordinate system used for the phase space. Starting from their representations in the laboratory phase space coordinate systems, we can "pullback" the distribution functions or the moment forms to express the moment integrals in an arbitrary new coordinate system, which can be non-canonical and non-fabric.

This general construction has been applied to the pullbacks of the guiding center transformation and the gyrocenter transformation. It has been demonstrated that the systematic 
treatment of the moment integrals provided by the pullback transformation is an essential component of the gyrokinetic theory itself. Without such a systematic treatment, the gyrokinetic theory is incomplete and many important physics features, such as the gyrokinetic equilibrium and the compressional Alfven wave, can not be readily recovered. Illustrative examples have been discussed in Sec. III and Sec. IV.

\section{Acknowledgments}

This research was supported by the U.S. Department of Energy under contract AC02$76 \mathrm{CH} 03073$.

[1] R. G. Littlejohn, J. Math. Phys. 20, 2445 (1979)

[2] R. G. Littlejohn, Phys. Fluids 24, 1730 (1981).

[3] R. G. Littlejohn, J. Plasma Phys. 29, 111 (1983).

[4] W. W. Lee, Phys. Fluids 26, 556 (1983).

[5] D. H. E. Dubin, J. A. Krommes, C. Oberman, and W. W. Lee, Phys. Fluids 26, 3524 (1983).

[6] L. Chen and S. T. Tsai, Phys. Fluids 26, 141 (1983).

[7] L. Chen and S. T. Tsai, Plasma Phys. 25, 349 (1983).

[8] S. C. Yang and D. I. Choi, Phys. Lett. 108 A, 25 (1985).

[9] T. S. Hahm, Phys. Fluids 31, 2670 (1988).

[10] T. S. Hahm, W. W. Lee, and A. Brizard, Phys. Fluids 31, 1940 (1988).

[11] A. J. Brizard, J. Plasma Phys. 41, 541 (1989).

[12] A. J. Brizard, Phys. Fluids B 1, 1381 (1989).

[13] H. Sugama, Phys. Plasmas 7, 466 (2000).

[14] W. W. Lee, J. Comput. Phys. 72, 243 (1987).

[15] W. W. Lee and W. M. Tang, Phys. Fluids 31, 612 (1988). 
[16] S. E. Parker, W. W. Lee, and R. A. Santoro, Phys. Rev. Lett. 71, 2042 (1993).

[17] J. C. Cummings, Ph. D. Dissertation (Princeton University, 1995).

[18] A. M. Dimits, T. J. Williams, J. A. Byers, and B. I. Cohen, Phys. Rev. Lett. 77, 71 (1996).

[19] R. D. Sydora, V. K. Decyk, J. M. Dawson, Plasma Phys. Control. Fusion 38, A281 (1996).

[20] Z. Lin, T. S. Hahm, W. W. Lee, W. M. Tang, and R. B. White, Science 281, 1835 (1998).

[21] H. Qin, W. M. Tang, and G. Rewoldt, Phys. Plasmas 5, 1035 (1998).

[22] H. Qin, Ph. D. Dissertation (Princeton University, 1998).

[23] H. Qin, W. M. Tang, W. W. Lee, and G. Rewoldt, Phys. Plasmas 6, 1575 (1999).

[24] H. Qin, W. M. Tang, and G. Rewoldt, Phys. Plasmas 6, 2544 (1999).

[25] H. Qin, W. M. Tang, G. Rewoldt, and W. W. Lee 7, 991 (2000)

[26] H. Qin, W. M. Tang, and W. W. Lee, Phys. Plasmas 7, 4433 (2000).

[27] W. M. Tang, Nuclear Fusion 18, 1089 (1978). 


\section{External Distribution}

Plasma Research Laboratory, Australian National University, Australia

Professor I.R. Jones, Flinders University, Australia

Professor João Canalle, Instituto de Fisica DEQ/IF - UERJ, Brazil

Mr. Gerson O. Ludwig, Instituto Nacional de Pesquisas, Brazil

Dr. P.H. Sakanaka, Instituto Fisica, Brazil

The Librarian, Culham Laboratory, England

Mrs. S.A. Hutchinson, JET Library, England

Professor M.N. Bussac, Ecole Polytechnique, France

Librarian, Max-Planck-Institut für Plasmaphysik, Germany

Jolan Moldvai, Reports Library, MTA KFKI-ATKI, Hungary

Dr. P. Kaw, Institute for Plasma Research, India

Ms. P.J. Pathak, Librarian, Insitute for Plasma Research, India

Ms. Clelia De Palo, Associazione EURATOM-ENEA, Italy

Dr. G. Grosso, Instituto di Fisica del Plasma, Italy

Librarian, Naka Fusion Research Establishment, JAERI, Japan

Library, Plasma Physics Laboratory, Kyoto University, Japan

Research Information Center, National Institute for Fusion Science, Japan

Dr. O. Mitarai, Kyushu Tokai University, Japan

Library, Academia Sinica, Institute of Plasma Physics, People's Republic of China

Shih-Tung Tsai, Institute of Physics, Chinese Academy of Sciences, People's Republic of China

Dr. S. Mirnov, TRINITI, Troitsk, Russian Federation, Russia

Dr. V.S. Strelkov, Kurchatov Institute, Russian Federation, Russia

Professor Peter Lukac, Katedra Fyziky Plazmy MFF UK, Mlynska dolina F-2, Komenskeho Univerzita, SK-842 15 Bratislava, Slovakia

Dr. G.S. Lee, Korea Basic Science Institute, South Korea

Institute for Plasma Research, University of Maryland, USA

Librarian, Fusion Energy Division, Oak Ridge National Laboratory, USA

Librarian, Institute of Fusion Studies, University of Texas, USA

Librarian, Magnetic Fusion Program, Lawrence Livermore National Laboratory, USA

Library, General Atomics, USA

Plasma Physics Group, Fusion Energy Research Program, University of California at San Diego, USA

Plasma Physics Library, Columbia University, USA

Alkesh Punjabi, Center for Fusion Research and Training, Hampton University, USA

Dr. W.M. Stacey, Fusion Research Center, Georgia Institute of Technology, USA

Dr. John Willis, U.S. Department of Energy, Office of Fusion Energy Sciences, USA

Mr. Paul H. Wright, Indianapolis, Indiana, USA 
The Princeton Plasma Physics Laboratory is operated by Princeton University under contract with the U.S. Department of Energy.

\author{
Information Services \\ Princeton Plasma Physics Laboratory \\ P.O. Box 451 \\ Princeton, NJ 08543
}

Phone: 609-243-2750

Fax: 609-243-2751

e-mail: pppl_info@pppl.gov

Internet Address: http://www.pppl.gov 